\title{
Cristina di Svezia: una protagonista del Seicento, un enigma endocrinologico
}

\author{
Massimo Mannelli ${ }^{1}$
}

Accettato: 21 aprile 2021 / Pubblicato online: 6 agosto 2021

(c) The Author(s) 2021

\section{La vita}

Cristina di Svezia nacque nel dicembre del 1626, in un secolo freddissimo, in un paese freddissimo e ancora arretrato socialmente e culturalmente, anche se in piena trasformazione per merito di suo padre, Gustavo Adolfo detto il Grande, della dinastia Vasa e del suo principale consigliere, Axel Oxestierna, che fu anche il precettore di Cristina durante la sua infanzia. Gustavo Adolfo, grande guerriero e protagonista della guerra dei 30 anni, difensore del protestantesimo, portò la Svezia in primo piano nelle vicende Europee, rendendola una potenza militare grazie a profonde riforme nella struttura del suo esercito, favorendo la crescita economica attraverso lo sviluppo dell'industria mineraria e della produzione agricola. Morì in battaglia nel 1632, a soli 38 anni, lasciando Cristina, la sua unica figlia, erede all'età di 6 anni. La madre di Cristina, Maria Eleonora, principessa del Brandeburgo, innamorata del marito, per amore del quale aveva accettato la vita spartana di Stoccolma, non accettò la morte di Gustavo Adolfo e quasi uscì di senno, impedendo per più di un anno la sepoltura del marito per averlo ancora vicino.

Il suo rapporto con Cristina fu sempre conflittuale, caratterizzato da un attaccamento morboso e da un affetto mai corrisposto. Nelle sue memorie Cristina scrive: "la regina non mi poteva sopportare perché diceva che ero piccola e brutta".

Fin dalla infanzia, Cristina mostrò un carattere molto forte, un'intelligenza viva, una predisposizione allo studio dettata dalla sua curiosità ma anche una personalità piena di contrasti, contraddizioni e atteggiamenti sorprendentemente anticonformisti.

$\triangle$ M. Mannelli

massimo.mannelli@unifi.it

1 Dipartimento di Scienze Biomediche Sperimentali e Cliniche "Mario Serio", Università di Firenze, Firenze, Italia
Amava lo studio così come l'attività sportiva. Aveva una corporatura minuta ma la vigoria fisica di suo padre. Da lui aveva ereditato un carattere sanguigno, con crisi emotive, accompagnate da pianti e eccessi d'ira [1]. Nonostante la vigoria fisica, soffriva frequentemente di periodi di astenia, specie dopo aver contratto malattia o a seguito di periodi di intenso stress emotivo.

Lei stessa ci rivela che aveva un'avversione e un' antipatia invincibili per tutto quello che fanno e dicono le donne. I loro abiti, ornamenti e maniere le erano intollerabili; non aveva nessuna cura del suo incarnato, della sua figura e del resto della sua persona; non portava mai cuffia, né velo, raramente guanti. Disprezzava tutto ciò che era appannaggio del suo sesso. Non sopportava i vestiti lunghi e voleva indossare solo gonne corte. Per di più aveva un'incapacità irrimediabile per tutti i lavori manuali femminili [1].

Perfino i suoi giocattoli erano quelli tipici dei maschi. Si dilettava con pezzi di piombo che disponeva in formazione di combattimento per imparare le manovre militari. Aveva passione per le armi, i cannoni, le spade e tutto ciò che era militare. Le piaceva vivere all'aperto e aveva una passione per gli animali, specie cani e cavalli [1]. A Cristina piaceva la caccia, era una bravissima tiratrice e un'abilissima cavallerizza. A fronte di tutto ciò, Cristina ebbe innumerevoli interessi culturali, spese vere fortune per comprare manoscritti e quadri, studiò e imparò diverse lingue, anche antiche, ebbe interesse per il teatro e la danza, invitò a corte numerosi intellettuali, tanto che Stoccolma si meritò l'appellativo di Atene del Nord. Il suo interesse per la filosofia la spinse a invitare a corte Cartesio che, riluttante anche perché intimorito dal clima di Stoccolma, dovette cedere alla caparbietà di Cristina che, una volta avutolo a corte, lo circondò di tutte le attenzioni ma lo costrinse, lui che aborriva il freddo e amava dormire fino a tardi, a tenerle lezioni di filosofia alle 5 del 
mattino così che lo sfortunato filosofo contrasse una brutta polmonite che lo portò a morte.

La sua vita affettiva non fu priva di contrasti: ebbe relazioni affettive sia maschili che femminili, anche se non è chiaro fino a che punto si siano spinti tali rapporti. Di sicuro, per sua stessa ammissione, il grande amore della sua vita è stata una dama di corte, Ebba Sparre, detta Belle, che Cristina presentò all'ambasciatore inglese come sua compagna di letto.

Un punto su cui Cristina mai cedette benché fortemente sollecitata più volte dal parlamento, dai suoi parenti e dai suoi consiglieri, fu il suo matrimonio. Lo rifiutò con decisione a più riprese dicendo "mi è impossibile sposarmi. Ne sono assolutamente certa".

Il suo carattere ribelle e imprevedibile la condusse alla sconcertante decisione di abdicare, all'età di 25 anni, in favore del cugino Carlo Gustavo nonostante il parere contrario di tutti. Allo stesso tempo, dichiarò la sua conversione al Cattolicesimo, assumendo il nome di Cristina Alessandra (in onore di papa Alessandro VII) Maria (nome che mai usò nelle sue firme autografe) ma non fu mai chiaro se per convinzione o calcolo, avendo in programma, cosa che fece, di trasferirsi a Roma. Di sicuro non si mostrò mai una cristiana devota o di animo pio, arrivando anche a condannare a morte un suo segretario, il marchese Monaldeschi, accusato di averla tradita. Si circondò sempre di personaggi equivoci, avventurieri alla ricerca di facili guadagni. Prima e dopo essersi stabilita a Roma, viaggiò per l'Europa trattenendosi per lungo tempo a Parigi e Amburgo. Nonostante avesse abdicato, si considerò sempre una regina degna di tale titolo $\mathrm{e}$ non esitò a contrapporsi a re e papi.

Cercò, con l'aiuto dichiarato, ma mai attuato, del Cardinale Mazzarino di divenire regina del Regno di Napoli. Scandalizzò a più riprese nobili e cardinali con i suoi atteggiamenti anticonformisti, talora sconvenienti e imbarazzanti. Trascorse gli ultimi anni della sua vita a Roma nel palazzo Riario, circondandosi di musicisti e attori di commedia. Ospitò grandi artisti dell'epoca come Bernini, Scarlatti, Corelli. A Roma ebbe una lunga relazione affettiva con il Cardinale Azzolino ma non è chiaro se solo platonica.

L'ultima descrizione di Cristina è dovuta a Francois Maximilian Misson, scrittore e viaggiatore francese, che la incontrò a Roma nella primavera del 1688 e così la descrisse: "Ha più di sessant'anni, è di piccola statura, troppo grassa $\mathrm{e}$ corpulenta. Il suo complesso, la voce ed il volto sono quelli di un uomo. Ha un naso grande, grandi occhi blu, sopracciglia bionde ed un doppio mento con alcuni peli di barba. Il suo labbro superiore si sporge di poco. I suoi capelli sono color nocciola chiaro, e sono lunghi appena un palmo di mano; li porta dritti e non acconciati. Sorride spesso. Sarà difficile immaginarvi i suoi vestiti: una giacca da uomo, di satin nero, che le raggiunge le ginocchia, tutta abbottonata, con una maglietta nera molto corta, e scarpe da uomo, assieme ad una serie di nastri neri a mo' di cravatta, il tutto accompagnato da una cintura nera stretta allo stomaco che ancor più rivela le sue rotondità".

Morì il 19 aprile del 1689 e fu inumata, unica donna assieme a Matilde di Canossa, in San Pietro. Subito dopo la sua morte, gli artisti del suo circolo fondarono l'Accademia dell'Arcadia che molto influenzò la letteratura italiana del Settecento.

\section{L'enigma}

Cristina fu certamente un personaggio "ingombrante" del Seicento, pieno di contrasti e di contraddizioni, anticonformista, ribelle, amante della libertà, dotata di un ego smisurato che pose sempre avanti a tutto.

C'è una spiegazione medica che giustifichi le caratteristiche di questo personaggio unico nel suo tempo?

Alla nascita Cristina fu dichiarata di sesso maschile dalle levatrici, presentando un'ipertrofia clitoridea che non doveva essere lieve. Solo il giorno seguente, un esame più accurato permise di capire che era una femmina. Non è chiaro a quale stadio di Prader si presentassero i suoi genitali esterni, ma certo l'androgenizzazione non doveva essere banale. Che Cristina fosse geneticamente una femmina è quanto si desume dall'esame condotto sui suoi resti nel 1965 dall'antropologo svedese Carl-Herman Hjortsjö, il quale non trovò "tracce di una intersessualità della regina, ovvero del fatto che fosse dotata sia di organi genitali maschili che femminili" [2]. Nel 1965 l'endocrinologia, così come la genetica, erano branche ai primordi e niente di più sembra sia dato di sapere. Il suo sesso femminile, del resto, lo si sarebbe potuto desumere anche dal fatto che Cristina presentò flussi mestruali, non si sa quanto regolari, ma almeno in qualche occasione, scarsi e dolorosissimi. Che la causa possa essere stata un ematocolpo o un addirittura un ematometra per la ridotta pervietà del canale vaginale? E quel suo ostinato rifiuto del matrimonio non sarà stato determinato anche dalla consapevolezza della sua anomalia genitale? Per spiegare la sua avversione, ella stessa dichiarò al Parlamento svedese "Vi dico ora che mi è impossibile sposarmi. Ne sono assolutamente certa. Non intendo spiegarvi i motivi". Il tono androgenico elevato può certamente aver influito sul suo orientamento psicosessuale, inducendo il suo carattere aggressivo, i suoi atteggiamenti maschili nel vestire, nel camminare e nel parlare talora in modo sboccato, giustificando la sua riferita forza muscolare e i suoi affetti omosessuali. Certamente ebbe effetto sulla sua bassa statura (era alta 150 $\mathrm{cm}$ ), che contrasta con la normale statura della madre tedesca e con quella del padre Gustavo Adolfo che, a detta di un inviato olandese, era molto alto e a cui assomigliava moltissimo, a cominciare dai capelli biondi, dai tratti del volto e soprattutto dal profilo prominente del naso lungo e affilato (Fig. 1). Anche la sua voce fu definita "profonda ed aspra", in accordo con l'ipotesi di un iperandrogenismo. 
Fig. 1 (a) Ritratto di Cristina di Svezia di Sébastien Bourdon (1653, olio su tela $72 \times 58 \mathrm{~cm}$, Nationalmuseum, Stoccolma). I tratti fisionomici della giovane Regina ritratta all'età di circa 25 anni appaiono ingentiliti e idealizzati, al contrario di quanto rilevabile dalla presumibilmente contemporanea incisione di Claude Quillet (1602-1661), riprodotta in $(\mathbf{b})$
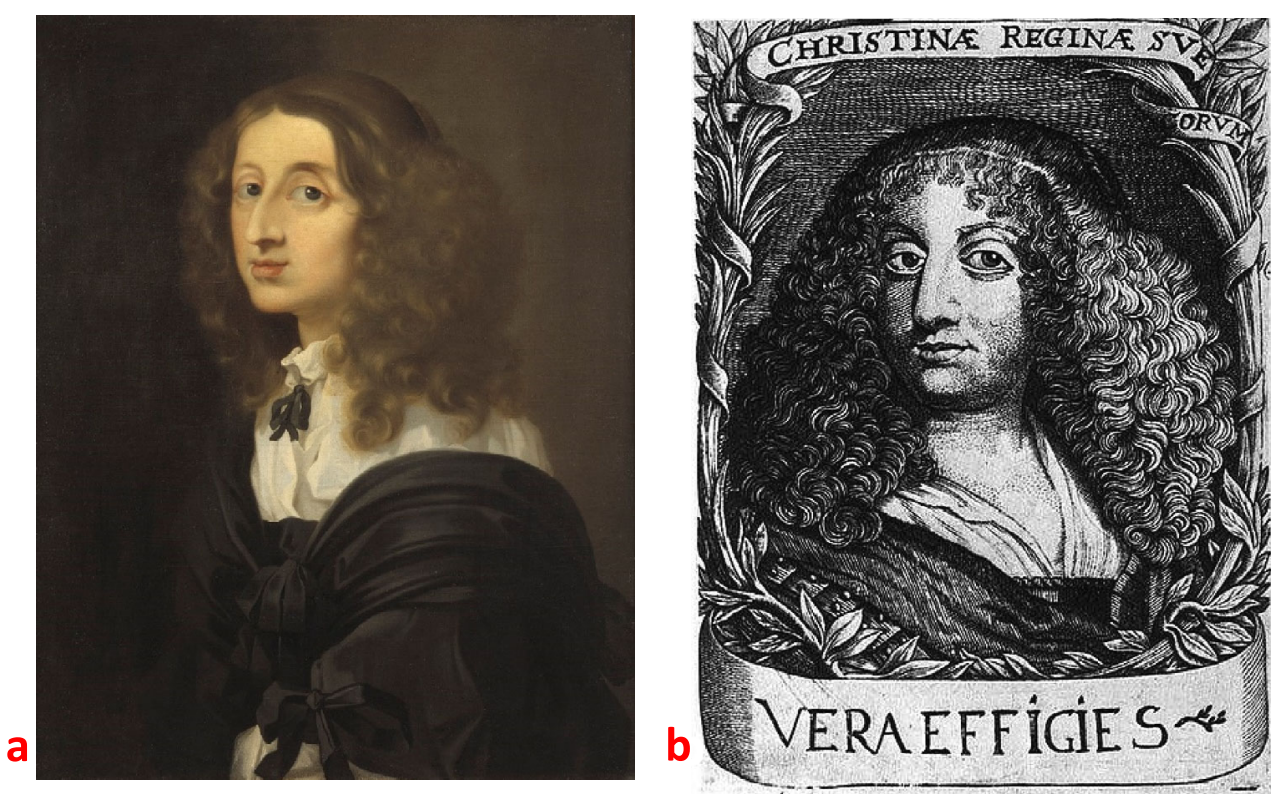

Quale può essere stata la causa di questo iperandrogenismo? La natura congenita e la storia clinica di Cristina sembrano orientare per una patologia surrenalica, probabilmente un deficit di 21-idrossilasi.

È interessante notare che prima di Cristina, la madre Maria Eleonora aveva avuto altre tre gravidanze: nel $1621 \mathrm{Ma}-$ ria Eleonora partorì una figlia nata morta, nel 1623 partorì una bambina, Cristina, che visse solo 11 mesi, e nel 1625 diede alla luce un figlio nato morto. Niente sappiamo delle caratteristiche di questi figli ma è possibile ipotizzare, in via teorica, che alla base di qualcuno di questi precocissimi decessi possa esserci stata un'insufficienza surrenalica.

Curiosamente, la madre Maria Eleonora, che nei primi anni non mostrò affetto per la figlia, definì la piccola Cristina come brutta e "di pelle scura come un piccolo moro". Tutto questo è in netto contrasto con i capelli biondi e gli occhi azzurri di Cristina. Che fosse una melanodermia da cronico aumento compensatorio di ACTH? Questo tratto sembra essere stato notato anche molti anni dopo quando, durante un soggiorno della regina in Francia, la contessa di Montpensier osservò che Cristina si metteva "una grande quantità di cipria e di crema per il viso" che rendeva la sua pelle bianca mentre pochi giorni dopo la sua pelle sarebbe risultata decisamente più scura [1].

Infine, se l'affezione era un deficit di 21-idrossilasi, era accompagnata da perdita di sali? A dispetto della vigoria fisica, Cristina ha sofferto, specie nell'infanzia e in gioventù, di episodi di profonda astenia, specie in occasioni di febbri, di svenimenti e di stati di profonda prostrazione soprattutto in occasione di susseguirsi di eventi emotivamente per lei molto stressanti. Dopo un periodo stressante per l'ostruzionismo che creò nei confronti del potente reggente Axel Oxenstierna, che l'aveva protetta e difesa dalla morte del padre alla maggiore età, si ammalò gravemente e ritenne di essere in pericolo di vita. Attribuì la malattia al grande sfinimento dovuto alla gestione degli affari di stato. Dopo una breve ripresa, Cristina cadde di nuovo malata ed ebbe anche una grave forma di morbillo [1].

Nel 1650, Cristina aveva 23 anni, durante un inverno particolarmente freddo, nel mese di marzo Cristina si ammalò nuovamente e si riprese dopo molte settimane.

Nel 1651, dopo settimane di violente discussioni relative al suo rifiuto di sposarsi, lo stress emotivo finì per ripercuotersi sulla salute della regina e una sera, a cena, Cristina si accasciò all'improvviso, sussurrò un addio e svenne rimanendo senza conoscenza per un'ora, con le pulsazioni così deboli da risultare quasi inavvertibili. Si riprese molto lentamente [1]. Tutto questo può suggerire che in occasioni particolarmente stressanti e protratte il compenso surrenalico possa essere stato insufficiente.

Ma queste sono tutte supposizioni. Oggi la genetica ci saprebbe svelare la verità.

\section{Storia del deficit di 21-idrossilasi}

L'associazione fra pseudoermafroditismo femminile e disfunzione surrenalica era già nota negli anni '30 del Novecento. Il quadro clinico di androgenizzazione dei genitali esterni e degli annessi cutanei veniva riportato in associazione con alterazioni della corticale delle ghiandole surrenaliche che andavano dall'iperplasia bilaterale alla presenza di una neoplasia [3].

Tale associazione veniva ribadita nel decennio successivo quando nei trattati di endocrinologia si introduceva il capitolo dello pseudoermafroditismo femminile da patologia sur- 
renalica caratterizzata da un'evidente iperplasia bilaterale della corticale [4].

Nei primi anni ' 50 vengono riportati casi familiari della sindrome congenita surrenalica che getta luce sul carattere ereditario e, pertanto, geneticamente determinato della sindrome [5] e si evidenzia come il trattamento con cortisone possa migliorare il quadro [6]. Nel decennio successivo, lo studio di pedigree familiari e la possibilità di misurare nelle urine metaboliti steroidei ad azione androgenica porta alla conclusione che l'iperplasia surrenalica virilizzante è causata da un errore della steroidogenesi surrenalica geneticamente determinato [7].

Nel 1968 Maria New pubblica un capitolo sul Pediatric Clinics of North America dove vengono descritti i vari difetti congeniti della steroidogenesi surrenalica e i quadri clinici correlati fra i quali quello nettamente più frequente riguarda la 21-idrossilasi [8].

Infine, nel 1986 viene pubblicata la sequenza del gene della 21-idrossilasi e del suo pseudogene, presenti nel cromosoma 6 [9]. Le più recenti novità nella diagnosi genetica della sindrome sono state di recente analizzate in questa Rivista [10].

Funding Note Open access funding provided by Università degli Studi di Firenze within the CRUI-CARE Agreement.

Conflitto di interesse L'autore Massimo Mannelli fa parte del Comitato di redazione e dichiara di non avere conflitti di interesse.

Consenso informato Lo studio presentato in questo articolo non ha richiesto sperimentazione umana.

Studi sugli animali L'autore di questo articolo non ha eseguito studi sugli animali.

Nota della casa editrice Springer Nature rimane neutrale in riguardo alle rivendicazioni giurisdizionali nelle mappe pubblicate e nelle affiliazioni istituzionali.
Open Access This article is licensed under a Creative Commons Attribution 4.0 International License, which permits use, sharing, adaptation, distribution and reproduction in any medium or format, as long as you give appropriate credit to the original author(s) and the source, provide a link to the Creative Commons licence, and indicate if changes were made. The images or other third party material in this article are included in the article's Creative Commons licence, unless indicated otherwise in a credit line to the material. If material is not included in the article's Creative Commons licence and your intended use is not permitted by statutory regulation or exceeds the permitted use, you will need to obtain permission directly from the copyright holder. To view a copy of this licence, visit http://creativecommons.org/licenses/by/4.0/.

\section{Bibliografia}

1. Bukley V (2004) Christina Queen of Sweden. The restless life of a European eccentric. Harper Collins Publisher Inc, New York

2. Hjortsjö C-H (1966) Drottning Christina. Gravöppningen i Rom 1965 En kulturhistorisk och medicinsk-antropologisk undersökning. Bokförlaget Corona, Lund

3. Brugsch T (1931) Trattato di medicina interna, trad it. UTET, Torino, p 203

4. Pende N (1949) Endocrinologia. Patologia e clinica delle ghiandole a secrezione interna. Società Editrice Libraria, Milano, pp 483-486

5. Bentinck RC, Hinman FSr, Lisser H, Traut HF (1952) The familial congenital adrenal syndrome: report of two cases and review of the literature. Postgrad Med 11:301

6. Wilkins L (1952) The diagnosis of the adrenogenital syndrome and its treatment with cortisone. J Pediatr 41:860

7. Childs B, Grumbach MM, Van Wyk JJ (1956) Virilizing adrenal hyperplasia; a genetic and hormonal study. J Clin Invest 35:213-222

8. New MI (1968) Congenital adrenal hyperplasia. Pediatr Clin N Am 15:395

9. White PC, New MI, Dupont B (1986) Structure of human steroid 21-hydroxylase genes. Proc Natl Acad Sci USA 83:5111-5115

10. Menabò S (2021) Novità nella diagnosi genetica di iperplasia surrenale congenita da deficit della 21 idrossilasi. Endocrinologo 22:103-108 